\title{
Performance factors in regard to impaired memory and tolerance induced by atropine sulfate
}

\author{
J. W. KASCKOW, G. J. THOMAS, and R. M. HERNDON \\ University of Rochester, Rochester, New York
}

\begin{abstract}
Acetylcholine is a widespread neurotransmitter in brain, but many studies have proposed a special sensitivity of memory consequent to disturbance of acetylcholine transmission. The present paper demonstrates that systemically administered atropine sulfate, an anticholinergic drug, appears to impair "representational" memory in a dose-related manner. Memory was indicated by discriminations made under positive reward when no critical sensory cues were available to the animal at the time of choice. By inference, the successful discriminations must have been made on the basis of memory traces. The paper also demonstrates that a marked tolerance to effects of the drug develops with repeated administration, but no conclusions are possible concerning the mechanism underlying the tolerance. However, it cannot be concluded unequivocally that the anticholinergic drug is selectively affecting representational memory, because response times (a performance factor) are similarly affected.
\end{abstract}

Acetylcholine, a neurotransmitter that is widely distributed in the mammalian brain, is believed to play an important role in behavior (see Russell, 1982) and in the more specific behavioral function of memory (see Bartus, Dean, Beer, \& Lippa, 1982). Many studies have shown that the anticholinergic drug scopolamine (e.g., Anisman \& Kokkinidis, 1975) has an especially disruptive effect on memory. Memory is often inferred from responses that were learned under aversively motivated circumstances, usually electric shock (Heise, 1981). Recently, it has been shown (Messer, Thomas, \& Hoss, 1983) that memory is impaired and tolerance to the drug scopolamine can occur after systemic injection (ip) or after intracerebral injections into hippocampus. In this study, memory was inferred from positively reinforced responses that were produced in a T-maze, that is, memory was derived from a paired-run procedure which presents the animal with a delayed-nonmatchingto-sample task; it is interpreted to indicate a higher order form of memory called representational memory (Thomas, in press).

Another anticholinergic drug, atropine sulfate, has also been shown to impair performance indicating memory. For example, Blozovski and Hennocq (1982) found impaired passive avoidance learning in young rats after systemic and intracerebral injections

The authors acknowledge support from Medical Scientist Training Grant T32 GM07356 to the first author and to Training Grant S-T32 MH14577 to the Center for Brain Research. Also, they are grateful to Mary Capozzi for assistance in preparing the manuscript. Requests for reprints should be sent to Garth J. Thomas, Center for Brain Research (Box 605), University of Rochester Medical Center, Rochester, NY 14642. of atropine. Also, Sutherland, Whishaw, and Regehr (1982) reported impaired performance on a spatial localization task in rats receiving atropine injections. This test took place in a Morris water maze in which successful performance depended on learning distal cues.

Studies with the anticholinergic drug atropine sulfate are complicated, especially when making crossspecies comparisons. Some animals (leaf eaters?) possess levels of a blood-liver enzyme, atropine esterase, that are high enough to decrease significantly the animals' reactivity to the drug (Weiner, 1980). For example, the LD-50 for rats is over $622 \mathrm{mg} / \mathrm{kg}$ of atropine sulfate according to the Merck Index (Windholz, 1976). Nonetheless, with sufficiently high doses, the drug does have anticholinergic effects.

The present study investigated the effects of systemic atropine sulfate on a positively rewarded response (delayed-nonmatching-to-sample), from which a relatively pure instance of representational memory that is substantially free from performance effects can usually be inferred. The first study established a dose-effect relationship, and the second demonstrated the tolerance that develops in respect to repeated tests and doses of the drug in terms of their deleterious effect on discriminative performance that indicates representational memory. We cannot conclude with certainty that the drug has its primary effect on memory, because speed of response (a performance factor) was similarly affected.

\section{Method}

Subjects. The experimental subjects were five young adult LongEvans male rats obtained from Blue Spruce Farms. They had a me- 
dian starting weight of $264 \mathrm{~g}$. Upon arrival, they were housed singly in wire-mesh cages and maintained on ad-lib food and water for 1 week for adaptation to the laboratory environment. Beginning on Day 7 , they were placed on a restricted, once-a-day feeding regimen that reduced their body weights to approximately $85 \%$ of their ad-lib weights in about a week. They were kept at that level of body weight throughout training and testing by supplementary feeding following their daily maze sessions.

Apparatus. The T-maze has been described before (Brito \& Thomas, 1981; Thomas, 1978). The essential characteristics of the maze are: (1) guillotine doors are located at the exit of the startbox (SB) and entrances to the goalboxes (GB); (2) the T-maze has no stem or arms, that is, the SB opens directly into the choice area and the right and left GBs open directly from opposite sides of the choice area; and (3) trays containing wet mash can be pushed into either GB to reward a correct response after the GB is entered. Thus, the sight or smell of food in the GB cannot guide the choice. A dim light suspended directly above the choice point of the Tmaze provided the only source of illumination in the test room.

Behavioral procedures. In order to habituate neophobic responses (exploratory tendencies, bouts of freezing and cautious wariness), the rats were first given 36 trials (6 per day) of one-door adaptation runs. In the adaptation runs, the rat was placed by hand into the SB, and the SB door was then raised, and a stopwatch was started. After a 2 -sec forced delay in the choice area, one or the other of the GB doors was raised (which door was raised depended on an irregular and balanced schedule). When the rat entered the only available GB, the door behind it was closed, the food dish was pushed in, and the watch was stopped. After a few seconds of eating while the score was being recorded, the rat was placed back into its home cage and the next rat was run. The process was repeated until all rats had completed six runs. By the end of the one-door adaptation sessions, all rats ran with alacrity (about $3 \mathrm{sec}$ per run, including the 2 -sec wait time in the choice area). They had learned that the maze was a safe place (no neophobic responses) and that there was food in the maze which could be obtained by locomoting to the distal end of the only available GB. However, they had had no learning experience as regards choice; the T-maze was essentially an L-shaped runway for them.

To get the rats to demonstrate representational memory, a tworun procedure was then added to the one-run procedure. The first run of a two-run trial was the same as the one-door run described above. The rat was forced to run to one or the other GB. This was called the "information run" (I). At the end of the eating period, the rat was placed back into the SB (instead of its home cage) and allowed a second run. This time, the doors to both GBs were raised simultaneously at the end of the 2-sec wait period and the animal made a choice as to which GB to enter (the $C$ run). If it chose correctly, that is, entered the GB opposite the one entered on the previous I run, it was rewarded by access to food for 8-10 sec before it was placed back into its home cage. If it chose incorrectly, it simply remained in the empty GB with no food for the same amount of time before it was removed to its home cage. The rats were run in squads of five, so that intertrial intervals were $3-4$ min long. The analogy with conventional delayed-nonmatchingto-sample is obvious. The forced, one-door run constitutes the sample. The succeeding two-door run requires that the rat remember which way it went (right or left) on the preceding I run in order to choose (discriminate) correctly and get the food reward. By the end of 10 days, 60 trials, 120 runs -6 trials per day-all rats were performing rapidly and consistently choosing correctly. Incidentally, nonmatching is easier than matching in this simple two-alternative situation, presumably because of rats' innate preference for alternation, but both are representational memory tasks (Stanton, Thomas, \& Brito, 1984).

Drugs. After preinjection training, tests were conducted to determine the effect of 5,10 , and $20 \mathrm{mg} / \mathrm{kg}$ of atropine sulfate. The drug was dissolved in distilled water and a control of physiological saline was also injected ip in the same volume (about $1 \mathrm{ml}$ ) on alternate sessions. The rats also received injections of methyl atropine nitrate $(20 \mathrm{mg} / \mathrm{kg})$. Each rat was tested for 12 trials under each condition of drug or saline in counterbalanced order. Note that each rat served as its own control. No rat was tested until at least $20 \mathrm{~min}$ had elapsed after injection, and all testing was completed within $3 \mathrm{~h}$ after injection. Sometimes, with the highest dose of atropine, an animal would not complete a run within 2 min. After a defaulted 1 run, the rat was removed from the maze and a time score of $120 \mathrm{sec}$ and an error for the usually following $\mathrm{C}$ run was recorded. After a defaulted $\mathrm{C}$ run, the rat obviously did not acquire a reinforcement, so an error with a response time of $120 \mathrm{sec}$ was recorded.

\section{Results}

Characteristically, in initial acquisition training, rats perform at a level of spontaneous alternation (or a little better), and individual differences are large. After completion of 60 trials, performance is usually crowded against the ceiling of $100 \%$ correct, and individual differences are small. That was clearly the case for this group of rats. The median percentage correct in the first 12 trials was $83 \%$. On the last 12 trials (Trials 49 through 60 ), one rat made one error. All the rest performed perfectly.

Delayed-nonmatching-to-sample in a T-maze is a simple task for rats. It is acquired rapidly (few trials) to a consistently high degree of accuracy. It should be pointed out that as each trial (pair of runs) involves trial-specific learning, the observed gradual improvement does not represent the gradual acquisition of an association between a sensory cue and a response, but, instead, indicates that the rats were simply learning to use their memory of the direction turned (or the GB entered) on an I run to guide their choice on the succeeding $\mathrm{C}$ run. Some rats discriminated at a statistically significant level (expanded binomial) from the beginning of training.

Figure 1 shows the effect of the drugs on representational memory, which is so called because, in order to choose correctly, the rat must discriminate a

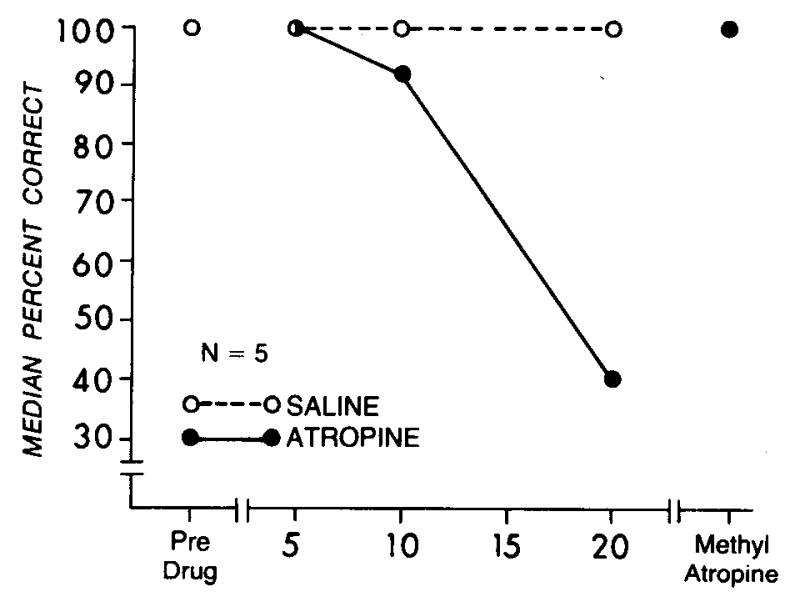

ATROPINE $(\mathrm{mg} / \mathrm{kg})$

Figure 1. Dose-effect curves showing the Increasing deleterious effect of increasing doses of atropine sulfate on memory (median percentage accuracy). The point to the far left indicates preinjection performance, and the point at the extreme right shows performance after a $20-\mathrm{mg} / \mathrm{kg}$ dose of methyl atropine nitrate. 
memorial representation (trace?) of cue stimuli that are no longer present to the animal's sensorium (stimuli from the I run). The figure presents a plot of the median percentage correct as a function of saline (open circles) and drug dosage (filled circles). Preinjection performance is indicated at the extreme left, and performance after injection of methyl atropine nitrate, which does not cross the blood-brain barrier and presumably has principally peripheral effects (Blozovski \& Hennocq, 1982), is shown at the extreme right side of the figure. Each rat received two six-trial sessions under each drug dose and saline condition, so values shown in the graph represent percentage correct in 12 trials.

The preinjection point shows that the rats were performing well against the ceiling of $100 \%$ accuracy. With saline, they continued to do so on alternate days throughout the study. However, after atropine sulfate injections, accuracy of performance decreased substantially. At the smallest dose $(5 \mathrm{mg} /$ $\mathrm{kg}$ ), the differences between the drug and saline conditions in the same rats were not significant. At doses of $10 \mathrm{mg} / \mathrm{kg}$, the differences were small but significant $(p<.05)$. (All reported $p$ values are based on quantiles of the Wilcoxon signed ranks test; Conover, 1971.) With doses of $20 \mathrm{mg} / \mathrm{kg}$, differences from saline runs were large and also significant $(p<.025)$. Obviously, methyl atropine nitrate, at least at the dose used, had no effect on choice accuracy.

However, the drug effect could not be unequivocally attributed to an effect on representational memory, since the drug also affected response times. Figure 2 plots median response times (seconds) for treated and control I runs and $C$ runs. The points to the left side of the figure indicate that response times were at a minimum of $3 \mathrm{sec}(2-\mathrm{sec}$ wait time in the choice area plus 1 -sec run time) at the end of preinjection training. (Times were measured to the nearest second.) The lowest dose of atropine and the

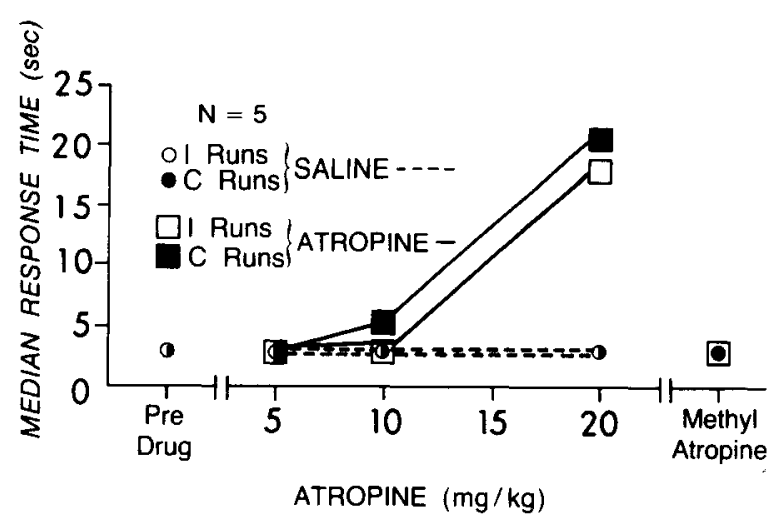

Figure 2. Curves depicting the effect on median response times of the doses of atropine sulfate. The points at the extreme left and extreme right show median response times of preinjection runs and of runs made after injections of methyl atropine nitrate, respectively. intermediate dose produced no marked change in response speeds. However, at the highest dose, there was a significant increase in response times for both $\mathrm{I}$ runs and $C$ runs (both ps $<.025$ ). The differences between I and $C$ runs were not significant. Injections of methyl atropine nitrate (extreme right side of the figure) had no effect on response times.

\section{TOLERANCE}

Observations of performance of individual rats under various combinations of treatments suggested that there might be a decided tolerance effect of the drug, that is, successive doses of the drug might yield less and less of a deleterious effect on representational memory. Therefore, a new group of animals was obtained to investigate this possibility.

\section{Method}

Subjects. A second batch of six Long-Evans rats of the same sex and similar weights and age as in the above study was obtained from the same dealer.

Apparatus. The T-maze used was the same as that described above.

Behavioral procedure. The preinjection procedures were the same as those described above.

Drug injections. To test for tolerance, a highly effective dose of $20 \mathrm{mg} / \mathrm{kg}$ atropine sulfate was used. Each rat was tested over two sessions (12 trials) for three times with drug and three times with saline on alternate days. All injections were administered systemically (ip). Half the rats received control saline and then drug. The other three animals received the treatments in reverse order. Also, as in the study reported above, all rats received $20 \mathrm{mg} / \mathrm{kg}$ methyl atropine nitrate on the final day of testing.

\section{Results}

The results are shown in Figure 3, which plots median percentage correct in 12-trial blocks as a function of the sequence of atropine sulfate injections. Performance under atropine is indicated by filled circles; performance under saline, by open circles. The point at the far left shows the median performance level during the last preinjection block of 12 trials $(92 \%)$. At the far right side of the figure, the median percentage correct achieved after injections of methyl atropine nitrate is shown. The results are clear-cut. Whenever the rats received saline, the median performance stayed consistently against the ceiling of $100 \%$ correct. On the other hand, the first two injections of atropine sulfate (in the same rats) reduced accuracy to levels that differed signigicantly from the saline performance (ps $<.01$ and .05 , respectively). The third injection of the drug had a nonsignificant effect on memory. The last point to the right side of the figure shows that methyl atropine nitrate, as in the earlier study, had no effect on performance.

Figure 4 shows marked performance effects, as indicated by time scores. In the 12-trial block of responses after the first injection of atropine, there is a marked increase in time scores for both I runs (open 


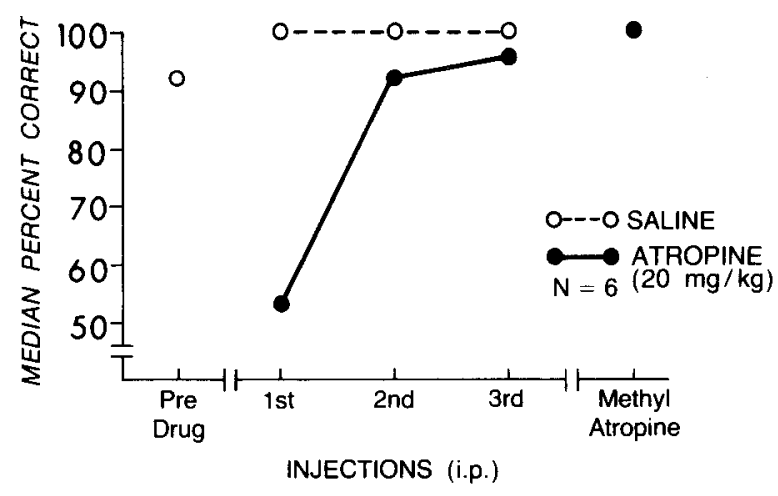

Figure 3. Median percentage correct as a function of physiological saline injections and of sequential doses of atropine sulfate $(20 \mathrm{mg} / \mathrm{kg})$. Preinjection level and the level after injection of methyl atropine nitrate are indicated by points at the extreme left and right sides of the figure.

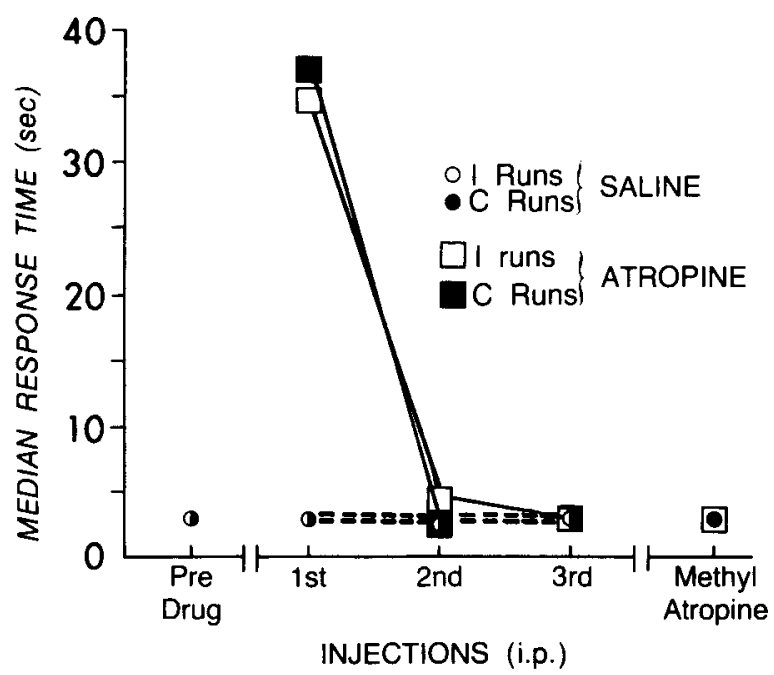

Figure 4. Median response times as a function of injections of physiological saline and sequential doses of atropine sulfate. Preinjection performance and performance under methyl atropine nitrate are indicated at the extreme edges of the figure.

squares) and $\mathrm{C}$ runs (filled squares) compared with performance after saline injections (both ps $<.01$ ). However, the median time scores on the second and third 12-trial blocks showed essentially the same response times as after saline-control injections. Methyl atropine nitrate had no effect on response times just as it had no effect on response accuracy. Again, there was no difference in response times between $I$ and $C$ runs.

\section{DISCUSSION}

The experimental findings described above indicate that the anticholinergic drug atropine sulfate appears to have a dose-related deleterious effect on the performance of a representational memory task indexed by a delayed-nonmatching-to-sample test under positive reward. A very rapid tolerance to the drug's effect on a memory-indicating performance develops with repeated doses. However, it is not entirely persuasive that the drug impairs memory or that memory becomes tolerant to the effects of the drug. The difficulty with concluding that the drug is primarily affecting memory comes from the fact that speed of responding, a nonassociative performance factor, is also affected.

The rats' slowness in responding, as shown in Figures 2 and 4, was not the result (as indicated by observations) of some gross impairment of motor coordination or impairment of general alertness (consciousness). Instead, the drug seemed to enhance general alterness and produced a return to cautious wariness, charateristic of naive animals in the maze. They were cautious and tentative in their movements, and they actively sniffed at features of the maze in an exploratory way as they had not done since early trials of preinjection acquisition. Some of them even squeaked in response to a startling touch-a very rare response in well-gentled rats. Post hoc, these behavioral charcteristics could be attributed to amnesia for the previous habituation and adaptation to the maze and to handling, but it is just as plausible to speculate that the drug altered some performance factors, for example, they produced changes in temperament that play an important role in modulating the index discrimination from which remembering is inferred.

Much evidence indicates that acetylcholine is a widespread transmitter in mammalian brain. Its ubiquity is seen in acetylcholinesterase-stained sections in the stereotaxic atlas by Paxinos and Watson (1982). Admittedly, choline acetyltransferase would be a more precise indicant, as seen in the atlas by Kimura, McGeer, Peng, and McGeer (1981). It seems plausible to suppose that systemic administration of anticholinergic drugs such as atropine sulfate could result in partial disconnection of complex patterns of synaptic activation which would result in very altered function. The altered brain function as inferred from behavior, could show up as a very "strange" and novel world as it is represented by patterns of brain activity mediating stimuli and responses and their interactions. Atropine has been shown to cause disorientation, hallucinations, and delirium in humans (Weiner, 1980).

Small, more or less discrete brain lesions in the posterior septum (Stanton et al., 1984; Thomas \& Spafford, 1984) in prelimbic cortex (area 32) (Thomas \& Spafford, 1984) in olfactory bulbs (Yu \& Wong, 1980), in nucleus basalis magnocellularis (Ordy, Thomas, Dunlap, \& Blosser, 1983), in mammillothalamic tracts (Thomas \& Gash, 1984), and probably in other places, all impair representational memory. These observations suggest that the capability for representational memory is widespread in brain and not dependent on a single anatomical system of now known cell groups and connections. That all brain is not involved is indicated by the fact that le- 
sions in dorsolateral frontal cortex of rat (Stanton et al., 1984), in anterior cingulate cortex (Thomas \& Brito, 1980), in dorsal hypothalamus just posterior to the paraventricular nuclei of hypothalamus (Thomas \& Gash, 1984), plus accidental lesions in anterior septum or dorsal midline thalamus (paraventricular nuclei of the thalamus) are all without effect on the accuracy of discriminations which indicates representational memory. However, all of the above listed positive brain sites stain heavily for acetylcholinesterase (or connect structures which do), so it is not surprising that anticholinergic drugs impair memoryindicating performances. Perhaps, intracerebral injections of anticholinergics into specific brain regions could have behavioral effects that would distinguish between general performance factors of systemic administration and more specific effects on subcircuits that mediate representational memory. Preliminary findings by Messer (personal communication) suggest that possibility. However, such specific, but temporary, chemically induced synaptic disconnections might not show anything much different from that shown by the permanent disconnections produced by experimental lesions.

The demonstration of behavioral tolerance to the drug as regards both general performance effects (time scores) and representational memory impairment (accuracy scores) indicate nothing as regards mechanism. The tolerance could be purely behavioral-habituation to the drug-altered "world" (a sort of state-dependent learning); it could reflect merely a heightened level of atropine esterase, that is, repeated doses could result in higher levels of this enzyme by enzyme induction; or tolerance could be induced by changes at the cellular level in terms of characteristics of receptor sites. Gray and Severs (1955), for example, observed atropine tolerance in isolated intestinal loops of dogs.

Finally, a caveat is in order. The dose-response curve, presented above, was determined by administration of the drug doses in the order 20,10 , $5 \mathrm{mg} / \mathrm{kg}$. The observed decrement in memory with increasing dose might be due, in part, to drug tolerance. We were not aware of the possible significance of the tolerance effect at the time the first study was done. It was thought that the long period between drug doses with interspersed control injections of saline represented adequate control for any cumulative or tolerance effects. Perhaps it does not-a practical consideration for research on the effects of anticholinergic drugs on higher mental processes (memory).

\section{REFERENCES}

Anisman, H., \& Kokkinidis, L. (1975). Effects of scopolamine, d-amphetamine, and other drugs affecting catecholamines on spontaneous alternation and locomotor activity in mice. Psychopharmacologia, 45, 55-63.
Bartus, R. T., Dean, R. L., Beer, B., \& Lippa, A. S. (1982). The cholinergic hypothesis of geriatric memory dysfunction. Science, 217, 408-417.

Blozovski, P., \& Hennoce, N. (1982). Effects of antimuscarnic cholinergic drugs injected systemically or into the hippocampusentorhinal area upon passive avoidance learning in young rats. Psychopharmacology, 76, 351-358.

Brito, G. N. O., \& Thomas, G. J. (1981). T-maze alternation, response patterning, and septo-hippocampal circuitry in rats. Behavioural Brain Research, 3, 319-340.

Conover, W. J. (1971). Practical nonparametric statistics. New York: Wiley.

Gray, G. W., \& Severs, M. H. (1955). In vivo observations on the nature of atropine tachyphylaxis exhibited by intestinal smooth muscle. Journal of Pharmacology and Experimental Therapeutics, 113, 319-331.

HE ise, G. A. (1981). Learning and memory facilitators: Experimental definition and current status. Trends in Pharmacological Science, 2, 158-160.

Kimura, H., McGeer, P. L., Peng, J. H., \& McGeer, E. G. (1981). The central cholinergic system studied by choline acetyltransferase immunocytochemistry in the cat. Journal of Comparative Neurology, 200, 151-201.

Messer, W. S., Jr., Thomas, G. J., \& Hoss, W. P. (1983). Behavioral tolerance to scopolamine in the rat using a working memory task. Society for Neuroscience Abstracts, 9, 826.

Ordy, M., Thomas, G., Dunlap, W., \& Blosser, J. (1983). Comparison of memory in rats after lesions in medial septal area and nucleus basalis magnocellularis. Society for Neuroscience Abstracts, 9, 639.

Paxinos, G., \& Watson, C. (1982). The rat brain in stereotaxic coordinates (Plate 10). New York: Academic Press.

Russell, R. W. (1982). Cholinergic system in behavior: The search for mechanism of action. In R. George, R. Okun, \& A. K. Cho (Eds.), Annual review of pharmacology and toxicology (pp. 435-463). Palo Alto, CA: Annual Reviews.

Stanton, M. E., Thomas, G. J., \& Brito, G. N. O. (1984). Posterodorsal septal lesions impair performance on shift and stay working memory. Behavioral Neuroscience, 98, 405-415.

Sutherland, R. J., Whishaw, I. Q., \& Regehr, J. C. (1982). Cholinergic receptor blockade impairs spatial localization by use of distal cues in the rat. Journal of Comparative and Physiological Psychology, 96, 563-573.

Thомаs, G. J. (1978). Delayed alternation in rats after pre- or postcommisural fornicotomy. Journal of Comparative and Physiological Psychology, 92, 1128-1136.

Thomas, G. J. (in press). Memory: Time binding in organisms. In L. Squire \& N. Butters (Eds.), Neuropsychology of memory. New York: Guilford Press.

Thomas, G. J., \& Brito, G. N. O. (1980). Recovery of delayed alternation in rats after lesions in medial frontal cortex and septum. Journal of Comparative and Physiological Psychology, 94, 808-818.

Thomas, G. J., \& GAsh, D. M. (1984). Mammillothalamic tracts and representational memory. Manuscript submitted for publication.

Thomas, G. J., \& Spaffond, P. S. (1984). Deficits for representational memory induced by septal and cortical lesions (singly and combined) in rats. Behavioral Neuroscience, 98, 394-404.

Weiner, N. (1980). Atropine, scopolamine and related antimuscarinic drugs. In A. G. Gilman, L. S. Goodman, \& A. Gilman (Eds.), The pharmacological basis of therapeutics (6th ed., pp. 120-137). New York: MacMillan.

Windholz, M. (Ed.) (1976). The Merck index (9th ed., p. 897). Rahway, NJ: Merck.

Yu, S., \& Wong, C. (1980). Septum and olfactory bulb lesions and dispositional and representational memory. Unpublished manuscript, University of Rochester.

(Manuscript received January 30, 1984; revision accepted for publication May 24, 1984.) 